DOI: $10.25283 / 2223-4594-2018-2-125-130$

УДК $551.326+626(005)$

\title{
О РЕГИСТРАЦИИ РИСКОВ ЛЕДОВОЙ НАВИГАЦИИ НА СЕВЕРНОМ МОРСКОМ ПУТИ
}

\author{
В. П. Трипольников \\ ГНЦ Арктический и антарктический научно-исследовательский институт \\ (Санкт-Петербург, Российская Федерация)
}

Статья поступила в редакцию 10 января 2018 г.

Рассмотрена проблема регистрации качественного состояния безопасности ледовой навигации на Северном морском пути. Даны методические рекомендации по проведению регистрации рисков. В качестве количественной оценки риска непредвиденной встречи с ледовым препятствием принята информационная энтропия сообщений о возможности появления ледовых препятствий. Проведены расчеты функций риска статистических моделей ледовых препятствий по классификации работы. Предлагается вариант сведе́ния к минимуму рисков непредвиденных встреч путем повышения качества мониторинга. Это позволит создать базу данных о рисках встречи ледовых препятствий по фиксированным маршрутам плавания как основу для оценки рисков. Применение рекомендаций может иметь перспективу в оценках и прогнозах материальных затрат на обеспечение безопасности ледового плавания.

Ключевые слова: лед, встреча, препятствие, вероятность, опасность, безопасность, сообщение, энтропия, риск.

\section{Ледовая обстановка - фактор риска на Северном морском пути}

Транспортировка углеводородов по Северному морскому пути (СМП) сопряжена с известными рисками экономических потерь и экологического ущерба [1]. Судоходство по СМП осложняется преодолением ледовых препятствий (ЛП), которые в непредвиденных обстоятельствах становятся потенциально опасными в отношении реализации экономических планов и состояния экологии. Современные исследования в обеспечение социально-экономического развития Арктической зоны Российской Федерации ориентированы на укрепление экологической и экономической безопасности хозяйственной деятельности. Чтобы отмечать прогресс в обеспечении безопасности переходов по СМП, надо фиксировать статус-кво фактора риска природной среды Арктики и регистрировать ступени его изменения.

Фактор риска количественно определяется произведением функции риска, выражающей вероятность неблагоприятного исхода в комплексе независимых совместимых случайных обстоятельств, на

() Трипольников В. П., 2018 последствия в ценовом выражении [2]. Неблагоприятный исход в виде неплановой задержки рейса [1] определится совмещением случайных угрожающих обстоятельств ледовой обстановки в данном переходе по СМП. Для успешного воплощения планов преодоления ЛП необходимы организационные решения, принятые на основе оптимизации в среднем вероятности неблагоприятного исхода [3; 4] и выбора актуальной метрической шкалы ЛП. Необходимо постоянно регистрировать в данной метрической шкале трудности ледовых переходов непосредственно в ходе морских транспортных операций. На основе опыта преодоления ЛП планируются последующие переходы в краткосрочной перспективе. Планирование рисков на срок более года, очевидно, требует совмещать результаты предшествовавших переходов с прогнозом на основе статистических данных мониторинга. Но долгосрочное планирование имеет смысл, если вероятность альтернативных исходов случайных событий отличается от 0,5. Следовательно, имеются проблемы эффективности и целесообразности прогноза, системного анализа рисков с целью их уменьшения, материальных и трудовых затрат на обеспечение безопасности 
ледового плавания. Решение этих проблем является задачей настоящего исследования.

\section{Метрическая шкала сложности ледового плавания и цена риска}

Различного вида ледовые препятствия преодолеваются разрушением льда, маневром обхода или выжиданием естественного освобождения пути. Затраты времени на преодоление отдельного ЛП

$$
\Delta t_{k}=t_{k}-t_{\mathrm{o}}
$$

где $t_{\mathrm{o}}$ - расчетное время перехода по чистой воде на дискретную единицу расстояния по маршруту; $t_{k}$ - фактическое время перехода при наличии ЛП; $k$ - индекс сложности преодоления.

Цена последствий в связи с ЛП при переходе по СМП равна произведению $\Delta t_{k}$ на стоимость фрахта в единицу времени. Негативные экологические последствия добавят к этой цене стоимость работ по рекреации или установленный штраф (детальное рассмотрение этого вопроса не входит в задачи данной статьи). Величина

$$
\sum \Delta t_{k}=\{t\}-|t|,
$$

где $\{t\}$ - длительность перехода с ледовыми препятствиями; $|t|$ - длительность перехода по чистой воде (исключая время плановых задержек), будет соответствовать сложности ледовой обстановки в совершенном переходе по СМП. Функция риска по свершившемуся факту ледового перехода: $\sum \Delta t_{k} /\{t\}=1-|t| /\{t\}$, статистическая вероятность встречи ЛП во времени определена в диапазоне от 0 до 1.

\section{Функции риска для прогностических моделей ледовой обстановки}

Реальная опасность аварии с экологическими последствиями возникает при внезапном появлении ЛП. Непредвиденные встречи с ЛП происходят, если средства мониторинга ледовой обстановки, обеспечивающие переход по СМП, оказываются неэффективными или неработоспособными, а караван или судно вынуждены двигаться вслепую. Известно также, что непредвиденные аномальные координаты ЛП в пространстве и времени создают иллюзию безопасности с негативными последствиями. Допускаем, что:

- события непредвиденных встреч с различными видами ЛП в разных пространственно-временны́х координатах на маршруте можно считать случайными, независимыми;

- максимальная повторяемость $r_{k}(\%)$ конкретного ЛП наблюдается локально, а $p_{k}=0,01 r_{k}$ - порядок квантиля $\Delta t_{k}$ этого вида ЛП на всем переходе по СМП;

- информационное сообщение (ИС) об опасности содержит статистические вероятности двух возможных исходов: опасного $p_{k}$ и безопасного $1-p_{k}$.

Для природного комплекса из $n$ видов ЛП по правилу дискретной оценки уровня потенциальной опасности возможно $N=2^{n}$ вариантов состояния, каждое из которых реализуется произведением не совпадающих по величине вероятностей исходов $k=1,2,3, \ldots, n$ случайного характера, включая и безопасные. В каждом проходе по СМП реализуется одно из состояний. Информационная энтропия (ИЭ) события встречи с отдельным ЛП

$$
S_{k}=-p_{k} \log _{2} p_{k}-\left(1-p_{k}\right) \log _{2}\left(1-p_{k}\right)
$$

выражает дефицит полученной информации (бит) относительно достоверного (с вероятностью 0 или 1) сообщения о нем, т. е. непредвиденность встречи с ЛП. ИЭ отдельного состояния природного комплекса $S_{N}=\sum S_{k}$ по $k=1,2,3, \ldots, n$. ИЭ всего природного комплекса максимальна при равновероятности всех состояний и равна $\log _{2} N$. Функция риска состояния [4]

$$
R_{N}=S_{N} / \log _{2} N=\left(\sum S_{k}\right) / n
$$

по шкале от 0 до 1 выражает среднюю по информационному содержанию непредвиденность встречи в любом состоянии комплекса ЛП. Если положить $R_{N}=-q \log q-(1-q) \log (1-q)$, то вероятности $q$, $(1-q)$ представляют ИС об опасности ожидаемого состояния комплекса для принятия решения. По условию $p_{k}<1-p_{k}$ меньшее значение $q<1-q$ также соответствует вероятности негативного (опасного) исхода. Тогда доминанта $(1-q)$ показывает вероятность безопасного исхода. По этим параметрам можно заранее задавать приемлемые границы риска субъективных решений с учетом независимых факторов рандомизации (игроков):

$$
q \leq p_{k}\left(1-P_{1}\right)\left(1-P_{2}\right),
$$

где $P_{1}$ - вероятность визуального наблюдения ледовой обстановки с борта судна; $P_{2}$ - вероятность работоспособности технического мониторинга.

Максимальный риск при $R_{N}=1$, характерный для отдаленного прошлого, соответствует принятию решения по жребию о возможности непредсказуемой встречи с ЛП при движении вслепую $\left(P_{1}=P_{2}=0\right.$, все $\left.p_{k}=0,5, q=0,5\right)$. Максимальный риск субъективного решения о возможности безопасного перехода по СМП при наличии только визуальных $\left(P_{2}=0\right)$ наблюдений с борта определяется двумя жребиями на удачный исход (по каждому виду ЛП $\left.p_{k}=0,5, P_{1}=0,5, q=0,25\right)$ и равен $R_{N}=0,811$ независимо от $n$. Если предусмотрено поступление оперативной информации о ледовой обстановке также и от средств технического мониторинга, то в прогнозе на отдаленное будущее максимальный риск субъективного решения о возможности безопасного 
Таблица 1. Сообщения о порядках квантилей опасных ледяных образований на СМП и соответствующая энтропия

\begin{tabular}{|c|c|c|c|c|c|c|c|}
\hline $\boldsymbol{k}$ & $\mathbf{1}$ & $\mathbf{2}$ & $\mathbf{3}$ & $\mathbf{4}$ & $\mathbf{5}$ & $\mathbf{6}$ & $\mathbf{7}$ \\
\hline $\mathrm{p}_{k}$ & $0,01-0,5-0,99$ & 0,07 & 0,016 & 0,1 & 0,001 & 0,005 & 0,001 \\
\hline$S_{k}$ & $0-1$ & 0,37 & 0,16 & 0,47 & 0,01 & 0,05 & 0,01 \\
\hline $\begin{array}{c}\sum S_{k}=1,07-2,07 ; \\
R_{N}=\sum S_{k} / 7=0,153-0,295\end{array}$ & & & & & & & \\
\hline
\end{tabular}

перехода ограничен тремя жребиями на удачный исход ( $\left.p_{k}=0,5, P_{1}=0,5=P_{2}, q=0,125\right)$ и равен $R_{N}=0,54$.

По результатам наблюдений в рейсе ИЭ дискретного распределения $\Delta t_{k}$ равна $S_{\Delta t}=\sum p_{k} \log _{2} p_{k}$. Функция риска реализованного состояния $R_{\Delta t}=S_{\Delta t} / \log _{2} n$, и можно выбрать варианты набора $p_{k}(1)$ или (2) для модельного представления $R_{N}=R_{\Delta t}$. Согласно эргодической гипотезе можно принять $\sum \Delta t_{k} /\{t\}=R_{\Delta t}=R_{N}$. Следовательно, можно прогнозировать потери времени на преодоление ЛП по моделям, в которых функция риска определена на основании статистических данных мониторинга ЛП.

\section{Модели рисков непредсказуемых встреч с ЛП по имеющейся информации о состоянии комплекса ЛП на переходе по СМп}

Имеем согласно [5] $n=22$ возможных вида ледовых препятствий судоходству по СМП, разделенных по происхождению на три группы: I - «опасные ледяные образования», II - «опасные ледовые явления», III - «неблагоприятные ледовые условия». Из этих трех групп можно образовать семь неповторяющихся моделей.

В табл. 1 выбраны порядки $p_{k}$ квантилей опасных ледяных образований (модель I) с учетом их относительной площади на обследованных акваториях и представлены результаты расчетов по формулам (1) и (2): 1 - поля и обломки полей многолетнего и двухлетнего льда, 2 - обломки полей и крупнобитый сильно торосистый лед, 3 - несяки, $4-$ зоны сильно сжатого и набивного льда, 5 - стамухи, 6 айсберги, 7 - обломки и куски айсбергов.

В первом столбце табл. 1 показаны интервалы, соответствующие возможному диапазону $p_{k}$. Например, сообщение о том, что поля и обломки полей многолетнего и двухлетнего льда покрывают 99\% площади акватории $\left(p_{1}=0,99\right)$, исключает непредвиденность: $S_{1} \approx 0$; при 50\%: $p_{1}=0,5, S_{1}=1$.

Шансы на исход встречи с ЛП этой модели при $R_{N} \leq 0,295$ в отношении опасность/безопасность $q /(1-q) \leq 0,05 / 0,95$, опасность от остальных групп ЛП не учитывается. Этот сокращенный вариант модели опасности ледовой обстановки можно применить при планировании технического оснащения судов.
Экстремальному состоянию опасности комплекса соответствует модель I + II + III с функцией риска $R_{N}=[2$ $, 07+(22-7) 0,54] / 22=0,462$, т. е. совместно с неопределенными исходами в остальных группах.

Выберем к модели II ситуации с «опасными ледовыми явлениями» в переходе по СМП не хуже, чем по 0,5-квантилям (табл. 2): 8 - обледенение судов и конструкций выносных терминалов, 9 - интенсивный дрейф льда и «ледовая река», 10 - сильное сжатие льда, 11 - внезапный вынос непроходимого льда на судоходную трассу (обвал), 12 - облипание корпуса. Из них события наружного обледенения судов и конструкций выносных терминалов, а также налипания льда ниже ватерлиний судов достоверно локализованы (обширны) по времени и по координатам, но случайны по интенсивности. Непредвиденная опасность этих событий происходит от превышения допустимого порога интенсивности и связанного с ним $\Delta t$. Здесь мы затрудняемся дать оценку пороговым значениям. Самый простой вариант - нулевой порог (принципиальная недопустимость угрожающей ситуации для конкретной техники) и, соответственно, равновероятность опасности исходов при любой интенсивности. Тогда ИЭ явлений по признаку $k$ (обледенения, облипания) $S_{k}=\log n_{k}$, а функция риска встречи с этими явлениями $R_{N}=\log n_{\mathrm{k}} / \log N_{k}$, где $n_{k}-$ число наблюдавшихся событий превышения порога; $N_{k}-$ число возможных событий (участников) за время наблюдений.

Более редкие опасные ледовые явления в виде аномально интенсивного дрейфа льда и «ледовой реки», аномально сильного сжатия льда, внезапного выноса непроходимого льда на судоходную трассу (обвала), по-видимому, имеют календарные периоды ( $T_{k}$-летние). При проблемах с работоспособностью средств наблюдения и оповещения $\left(P_{1}, P_{2}<1\right)$ ожидаемая максимальная функция риска $R_{N}$ состояния ледового плавания по этим опасным явлениям ограничен вероятностью опасного исхода $p_{k}=1 / T_{k}$, $q=0,25 / T_{k}$. Соответственно решение обратной задачи дает ожидаемые периоды $T_{k}$ по $k$ № $8,9,10$ - от полутора лет и более.

По значению $R_{N}$ в табл. 2 шансы на исход встречи с ЛП этой модели в отношении опасность/безопасность $q / 1-q=0,15 / 0,85$. Здесь риск выше, чем при 
Таблица 2. Условные сообщения о порядках квантилей опасных ледовых явлений на СМП и соответствующая энтропия

\begin{tabular}{|c|c|c|c|c|c|}
\hline $\boldsymbol{k}$ & $\mathbf{8}$ & $\mathbf{9}$ & $\mathbf{1 0}$ & $\mathbf{1 1}$ & $\mathbf{1 2}$ \\
\hline$p_{k}$ & 0,14 & 0,125 & 0,14 & 0,08 & 0,28 \\
\hline$S_{k}$ & 0,584 & 0,54 & 0,584 & 0,4 & 0,866 \\
\hline $\begin{array}{c}\sum S_{k}=2,974 ; \\
R_{N}=\sum S_{k} / 5=0,595\end{array}$ & & & & & \\
\hline
\end{tabular}

слепой проводке судов, так как практически исключается эффективность мониторинга. Эту сокращенную модель опасности можно применить для тестирования физических моделей опасных ледовых явлений.

Модель I + II имеет функцию риска опасного исхода в среднем по двум группам ЛП $R_{N}=(2,07+2,974) / 12=0,42$, которому соответствуют параметры мотивации для принятия решений: $1-q=0,915$ в пользу безопасного плавания, $q=0,085$ в пользу опасного исхода.

В модель III включены события ЛП, вызванные аномальными сроками ледообразования и очищения акватории ото льда, аномально тяжелыми условиями для ледовой проводки судов, всего в количестве 10. Их повторяемость, возможно, выявится в климатических циклах. Впредь до выяснения квантили этих видов непредвиденных опасностей следует принять с $p_{k}=0,125$, так как допускаются неопределенности (с вероятностями 0,5) возникновения ЛП, состояния технического мониторинга и визуального наблюдения. Значение функции риска модели III $R_{N}=0,54$.

Экстремальное состояние комплекса из 22 видов ЛП соответствует их совмещению в одном переходе по СМП (модель I + II + III), а функции риска (2) этого состояния

$$
R_{N}=(2,07+2,974+0,54 \cdot 10) / 22=0,475
$$

характеризует полученные сведения о комплексе по недостатку достоверной информации. За исключением данных из табл. 1 это означает, что в современную эпоху имеем незначительное положительное влияние результатов исследований ледовой обстановки на информационное описание опасных событий по сравнению с теоретической рандомизацией опасности ледового плавания по жребию.

\section{Коррекция рисков повышением качества мониторинга}

Задача мониторинга состоит в создании сообщений о потенциальной опасности с большей достоверностью, т. е. с вероятностями $p_{k}>>0,5$ или $p_{k}<<0,5$. Это приведет к очевидным последствиям как в тактике ледовых переходов, так и в принятии решений по планированию. При планировании возможных рисков на предстоящие переходы по СМП имеет смысл принимать начальные условия $P_{1}=P_{2}=0,5$. Повышением качества мониторинга ледовой обстановки можно задать параметры мотивации решения о предстоящем риске плавания до значений $1-q=0,99$ и $q=0,01$, соответственно $R_{N}=0,0808$, что согласно (3) потребует от мониторинговых систем выполнения условия $p_{k} \leq 0,25 q=0,0025$, т. е. практически достоверного сообщения. Положим, что среди $n=22$ на ближайшую навигацию останется $m$ возможных угрожающих факторов с вероятностью $p_{m}=0,5$, имеющих функцию риска $R_{m}=0,54$. Требуется определить качество мониторинга остальных $(n-m)$ угрожающих обстоятельств для сохранения заданного уровня параметров мотивации. На основании (2) и свойства аддитивности ИЭ запишем уравнение баланса:

$$
n R_{N}=(n-m) R_{n-m}+m R_{m} .
$$

В результате подстановок в (4) требуемых значений имеем:

- при $m=0$ достигается искомая функция риска $R_{N}=0,0808$ и $q=0,01$;

- при $m=1$ для сохранения $R_{N}=0,0808$ требуется повышение качества мониторинга остальных ИС и снижение их среднего риска до $R_{n-m}=0,059$, а вероятности ошибки до $q=0,007$;

- при $m=2$ требуется снижение риска до $R_{n-m}=0,035$, $q=0,0034$

- при $m>3$ требуется $R_{n-m}=0, q=0$, т. е. достоверная информация об остальных $(n-m)$ обстоятельствах. Вывод, естественно, один: надо уменьшать до нуля число не освещаемых мониторингом ЛП второй и третьей групп по классификации [5].

\section{Выводы}

Прогресс в обеспечении безопасности плавания по СМП может быть отмечен по результатам исследования рисков встреч с ЛП в рейсах и сопоставления фактических рисков с прогностическими моделями на основе статистических данных мониторинга.

Ключевым принципом для исследования сравнительных рисков комплексов случайных обстоятельств является использование понятия 
информационной энтропии, по определению учитывающее альтернативные исходы. ИЭ совершенного перехода по СМП следует рассчитывать по распределению статистических частот затрат времени на преодоление ледовых препятствий.

Среднее информационное содержание дефицита знаний (ИЭ) о непредвиденных встречах с ЛП в относительных единицах определяет функцию риска $R_{N}$ статистической модели.

Статус-кво природного комплекса ЛП на акватории СМП по состоянию знаний и успехов современного мониторинга описывается статистической моделью с функцией риска $R_{N}=0,475$, что незначительно отличается в лучшую сторону от модели жребия $\left(R_{N}=0,54\right)$. Уравнения баланса риска (4) позволяют рассчитывать варианты повышения качества мониторинга на основании требований безопасности и надежности принимаемых решений. Проблемным остается мониторинг предвестников труднопреодолимых и непреодолимых ледовых условий.

Идентификация реальных и модельных рисков создает возможность использования прогностических моделей для планирования материальных и трудовых затрат переходов по СМП.

\section{Литература}

1. Камаев Э. Л., Тарасов Ю. В. Особенности и риски морской транспортировки углеводородов в арктических условиях и возможные решения // Труды 7-й Международной конференции и выставки по освоению ресурсов нефти и газа российской Арктики и континентального шельфа стран СНГ (RAO / CIS Offshore 2005). 13-15 сентября 2005 года, СанктПетербург. - СПб.: Химиздат, 2005. - С. 61-66. 2. Добротворский А. Н., Яценко С. Я. Обеспечение безопасности проекта создания морского подводного газопровода на основе технологий сквозной оценки риска // Труды 7-й Международной конференции и выставки по освоению ресурсов нефти и газа российской Арктики и континентального шельфа стран СНГ (RAO / CIS Offshore 2005). 13-15 сентября 2005 года, Санкт-Петербург. - СПб.: Химиздат, 2005. - C. 441-445.

3. Гриняк В. М., Герасименко Л. В., Девятисильный А. С. Многоуровневая модель идентификации опасных ситуаций береговыми системами управления движением судов // Навигация и гидрография. - 2015. - № 40. - С. 29-38.

4. Лебедев Г. А., Трипольников В. П. Информационный анализ обстановки на акватории для определения качества мониторинга и риска столкновения ледяных образований с морским сооружением // Метеорология и гидрология. - 2012. - № 2. - С. 72-77. 5. Опасные ледовые явления для судоходства в Арктике / Под ред. Е. У. Миронова. - СПб.: ААНИИ, 2010. - 319 c.

\section{Информация о6 авторе}

Трипольников Владимир Петрович, кандидат физико-математических наук, старший научный сотрудник, Арктический и антарктический научно-исследовательский институт (199397, Россия, Санкт-Петербург, ул. Беринга, 38), e-mail: tvp80@aari.ru.

\section{Библиографическое описание данной статьи}

Трипольников В. П. О регистрации рисков ледовой навигации на Северном морском пути // Арктика: экология и экономика. — 2018. — № 2 (30). - C. 125-130. — DOI: 10.25283/2223-4594-2018-2-125-130. 


\title{
ON REGISTRATION OF ICE NAVIGATION RISKS ALONG THE NORTHEAST PASSAGE
}

\author{
Tripolnikov V. P. \\ State Research Center “Arctic and Antarctic Research Institute" (St. Petersburg, Russian Federation)
}

\begin{abstract}
The article is dedicated to the problem of registering the quality of ice navigation safety along the Northeast Passage and contains methodical recommendations for risk registration. Informational entropy of random events is accepted as quantitative estimate of risk of icy obstacles. Based on the work classification the calculations risk values of statistic models for icy obstacles is given. Options of minimum the risks of unpredicted encounters through improved accuracy of messages are discussed. These measures may allow to create a database of ice obstacle risks which can serve as a basis for navigation risks estimate. The recommendations can be implemented in estimates and forecasts of expenditures on safety in ice navigation.
\end{abstract}

Keywords: ice, encounter, obstacle, probability, danger, safety, communication, entropy, risk.

\section{References}

1. Kamaev E. L., Tarasov Yu. V. Osobennosti i riski morskoi transportirovki uglevodorodov $\mathrm{v}$ arkticheskikh usloviyakh i vozmozhnye resheniya. [The specifics and risks of sea transportation of hydrocarbons in arctic conditions and possible solutions]. Trudy 7-i Mezhdunarodnoi konferentsii i vystavki po osvoeniyu resursov nefti i gaza rossiiskoi Arktiki i kontinental'nogo shel'fa stran SNG (RAO / CIS Offshore 2005). 13-15 sentyabrya 2005 goda, Sankt-Peterburg. St. Petersburg, Khimizdat, 2005, pp. 61-66. (In Russian).

2. Dobrotvorskii A. N., Yatsenko S. Ya. Obespechenie bezopasnosti proekta sozdaniya morskogo podvodnogo gazoprovoda na osnove tekhnologii skvoznoi otsenki riska. [Safety provision for the project of building the submarine gas pipeline, based on the through risk estimate and control technoloigies]. Trudy 7-i Mezhdunarodnoi konferentsii i vystavki po osvoeniyu resursov nefti i gaza rossiiskoi Arktiki i kontinental'nogo shel'fa stran SNG (RAO / CIS Offshore 2005). 13-15 sentyabrya 2005 goda, Sankt-Peterburg. St. Petersburg, Khimizdat, 2005, pp. 441—445. (In Russian).
3. Grinyak V. M., Gerasimenko L. V., Devyatisil'nyi A. S. Mnogourovnevaya model' identifikatsii opasnykh situatsii beregovymi sistemami upravleniya dvizheniem sudov. [The multilevel model for the identification of dangerous situations with coastal systems for operation of shipping traffic]. Navigatsiya i gidrografiya, 2015, no. 40, pp. 29-38. (In Russian).

4. Lebedev G. A., Tripol'nikov V. P. Informatsionnyi analiz obstanovki na akvatorii dlya opredeleniya kachestva monitoringa i riska stolknoveniya ledyanykh obrazovanii s morskim sooruzheniem. [The informational analysis of situation in the sea region to assess the monitoring quality and to determine the risk of collision between ice formations and sea infrastructure]. Meteorologiya i gidrologiya, 2012, no. 2, pp. 72-77. (In Russian).

5. Opasnye ledovye yavleniya dlya sudokhodstva v Arktike. [Ice phenomena dangerous for shipping traffic in the Arctic]. Pod red. E. U. Mironova. St. Petersburg, AANII, 2010, 319 p. (In Russian).

\section{Information about the author}

Tripolnikov Vladimir Petrovich, Candidate of Physico-Mathematical Sciences, Senior Researcher, State Research Center "Arctic and Antarctic Research Institute" (38, Bering st., St. Petersburg, Russia, 199397), e-mail: tvp80@aari.ru.

\section{Bibliographic description}

Tripolnikov V. P. On registration of ice navigation risks along the Northeast Passage. Arctic: ecology and economy, 2018, no. 2 (30), pp. 125-130. DOI: 10.25283/2223-4594-2018-2-125-130. (In Russian). 